\title{
Long-range interactions and artificial poles
}

\author{
J. O. Stott, I. J. Thompson, J. A. Tostevin ${ }^{1}$ \\ ${ }^{1}$ Department of Physics, School of Physical Sciences, University of Surrey, \\ Guildford, Surrey GU2 5XH, United Kingdom
}

(Dated: November 5, 2011)

\begin{abstract}
The structure of the three-body continuum of the ${ }^{6} \mathrm{He}$ nucleus within the hyperspherical harmonic calculation scheme has been the subject of extensive study. An approximate treatment of the long-range couplings inherent in such models gives rise to unphysical poles in the deduced Smatrix at complex energies. We consider here a simple two-channel example, which includes long-range interactions, and demonstrates the existence of these artificial S-matrix poles. This is also illustrated in a physical context by considering the extreme adiabatic approximation of the hyperspherical harmonics technique for ${ }^{6} \mathrm{He}$. A correct treatment of the long-range $\left(1 / \rho^{\lambda}\right)$ behaviour of the channel potentials is shown to remove the artificial poles from the physically significant region of the complex energy plane.
\end{abstract}

PACS numbers: PACS numbers: 11.55.Bq, 25.70.Ef, 21.45.+v 


\section{INTRODUCTION}

Experiments with neutron-rich nuclear beams have confirmed that ${ }^{6} \mathrm{He}$ has a pronounced two-neutron halo which extends to large radii. This structure has been successfully modelled as a three-body system, with a $0^{+}$ground state, consisting of an alpha particle core and two neutrons forming the halo [1]. Recently, attention has focussed on the excited continuum structure of ${ }^{6} \mathrm{He}$, which has been probed experimentally using breakup reactions [2]. The low energy ${ }^{6} \mathrm{He} 2^{+}$resonance at about $1.8 \mathrm{MeV}$ excitation $[3,4]$, predicted by three-body models, has been experimentally observed however there has been no experimental signature of the higher $2^{+}$resonance predicted at about $3 \mathrm{MeV}$. Resonance positions for $0^{+}$and $1^{-}$ spin parity states have also been predicted [3], however these predictions have been criticized as being unphysical [5].

To compare experiment and theory it is important to correctly determine the position of the continuum resonances as predicted by theoretical models, according to the strict definition that a resonance corresponds to a pole of the S-matrix at a complex energy [6]. Our interest concerns long-range interactions which if truncated give rise to unphysical poles at complex energy. These are distinct from the redundant poles of Ma $[7,8]$ found in connection with the short-range exponential potential, and which are located on the positive imaginary $k$ axis, but are not bound states. A fuller discussion may be found in reference [9].

Within the hyperspherical harmonic calculation scheme the three-body Hamiltonians introduce long-range radial couplings. It has been shown in [10] that a radial truncation of such couplings gives rise to unphysical poles of the S-matrix, which were referred to in that article as artificial poles. In that work, integrations were carried out to large radii and so these unphysical poles were located very close to the origin of the complex energy plane. In the case of ${ }^{6} \mathrm{He}$, our interest is in using the R-matrix method to solve the large coupled channels sets in an interior region with a modest internal boundary radius. In this case we show that the artificial poles are located in the physically interesting part of the complex energy plane where we would wish to locate resonance poles.

In this work we first consider a simpler two-channel example where artificial poles are shown to exist if long-range interactions are truncated. These are shown to be removed when the long-range nature of the interaction is correctly incorporated. The physical implications are illustrated by considering the extreme adiabatic approximation of the hyperspherical 
harmonics technique in the case of the ${ }^{6} \mathrm{He}$ system.

\section{METHOD OF SOLUTION}

The expansion of the quantum mechanical three-body wave function in terms of the hyperspherical harmonic basis functions [4] reduces the Schrödinger equation to a system of coupled equations in one radial variable $\rho$, the hyper-radius, which is a measure of the overall size of the three-body system.

In this coordinate system the hyper-radial Schrödinger equations take the familiar form of a system of coupled two-body radial equations. The main difference is that, in the threebody case, the centrifugal barrier is $(K+3 / 2)(K+5 / 2) / \rho^{2}$ where $K$ is the hyperharmonic quantum number. Furthermore, the hyper-radial potentials are of a long-range nature, behaving asymptotically as inverse powers of $\rho$, despite the two-body interactions being of a short-range nature.

The R-matrix method [11] has been found to provide a numerically stable method for the numerical solution of these radial coupled channels equations with advantages over direct numerical integration of the system. This R-matrix method provides the means to evaluate the S-matrix at a boundary $\left(\rho=r_{m}\right)$ by matching to incoming and outgoing spherical waves. If it is assumed that all interactions can be neglected beyond the matching radius, then the functions used in the matching are simply diagonal Riccati-Hankel functions which are solutions of the free equation.

The internal R-matrix solution is most effective when using not too large values for the boundary radius $r_{m}$, otherwise large basis sets are required. We will show that it is then important to incorporate the long-range nature of the diagonal and coupling interactions correctly, and the matching must be done to solutions of the coupled radial equations in the asymptotic region $\left(\rho>r_{m}\right)$ where the interactions behave as sums of inverse powers of $\rho$. These solutions can be expressed as asymptotic series expansions in inverse powers of $\rho$ so it is advantageous to evaluate these at as large a $\rho$ as possible for faster convergence.

For their evaluation at complex energy this would suggest that complex coordinate rotation (or exterior rotation) [12-14] is necessary for the numerical calculation of the S-matrix. However we will show that it is still possible to calculate the S-matrix accurately over an appreciable region of the complex energy plane without using any form of coordinate rota- 
tion, and this enables the use of boundary radii appropriate to the R-matrix procedure. In this case however it is important to include the long-range nature of the potentials when matching at the R-matrix boundary.

\section{COUPLED CHANNELS POLES IN THE ADIABATIC LIMIT}

We know from [10] that one-channel solutions, calculated with radial truncation, have artificial poles. Artificial poles will also be present for any many channel calculation if longrange couplings are truncated. This fact is most easily demonstrated by considering a simple two-channel case.

In the limit of degenerate channel energies $E_{1}=E_{2}=E$ and equal diagonal and offdiagonal interactions, $V_{11}=V_{22}=V$ and $V_{12}=V_{21}=v$, it is well known that the two coupled equations

$$
\begin{aligned}
& (E-T-V) \phi_{1}=v \phi_{2} \\
& (E-T-V) \phi_{2}=v \phi_{1}
\end{aligned}
$$

can be decoupled. The $(2 \times 2)$ S-matrix, with elements $S_{i j}$ representing the coefficient of the outgoing wave in channel $i$ when there is a unit incoming wave in channel $j$, are obtained by adding and subtracting the Eqs. 1 giving rise to the two uncoupled equations for wave functions $\psi_{1}=\left(\phi_{1}+\phi_{2}\right) / 2$ and $\psi_{2}=\left(\phi_{1}-\phi_{2}\right) / 2$.

$$
\begin{aligned}
& (E-T-[V+v]) \psi_{1}=0 \\
& (E-T-[V-v]) \psi_{2}=0 .
\end{aligned}
$$

It follows that the diagonal and off-diagonal S-matrix elements $\left(S_{1}+S_{2}\right) / 2$ and $\left(S_{1}-S_{2}\right) / 2$ are linear combinations of the uncoupled one-channel S-matrices $S_{1}$ and $S_{2}$.

It may be further concluded that, in this adiabatic limit, any poles introduced by the truncation of $V$ or $v$ will be shared by the coupled channels set, which will see poles resulting from each truncated channel. This general feature is also to be expected in the non-adiabatic case. 


\section{INCLUSION OF LONG-RANGE COUPLINGS}

Given the above it is clearly important to include the long-range portion of the potential, while, for the use of the R-matrix interior solution, it is important to avoid numerically integrating to a large distance. Both these constraints can be satisfied, if we modify the functions that are used in the matching procedure. Instead of using the free incoming and outgoing waves solutions, functions must be used that are the actual solutions of the coupled equations in the presence of inverse power couplings. Various methods exist to generate such functions. In the following example the method of Rösel, as proposed in [15] for Coulomb excitation calculations, is applied. We assume the interactions behave asymptotically as a sum of inverse cubic and quintic powers of $r$. This asymptotic behaviour is that displayed by the hyper-radial couplings in the three body model. For a simple demonstration, a numerical example is considered where they are included in a two-channel case.

The radial Schrödinger equation is shown in Eq. 3 where $x=k r$ and the factor $2 m / \hbar^{2}$ has been absorbed into the asymptotic potential strengths $w_{j m}^{[3]}$ and $w_{j m}^{[5]}$,

$$
\left(\frac{d^{2}}{d x^{2}}+1-\frac{l_{j}\left(l_{j}+1\right)}{x^{2}}\right) \psi_{j}(x)=\sum_{m} \frac{k w_{j m}^{[3]} \psi_{m}(x)}{x^{3}}+\sum_{m} \frac{k^{3} w_{j m}^{[5]} \psi_{m}(x)}{x^{5}} .
$$

To perform the matching a complete set of coupled outgoing wave solutions can then be defined

$$
\psi_{j}^{t}(x)=a_{j}^{t}(x) \exp \left[i\left(x-\pi l_{j} / 2\right)\right]
$$

where the subscript labels the channel in which the outgoing waves appear as $x \rightarrow \infty$ and the superscript labels the channel in which there was an incoming wave. The asymptotic behaviour of $a_{j}^{t}(x)$ is such that $a_{j}^{t}(x) \rightarrow \delta_{j t}$ so that

$$
\psi_{j}^{t}(x) \longrightarrow \delta_{j t} \exp \left[i\left(x-\pi l_{j} / 2\right)\right]
$$

as the Eqs. 3 decouple as $x \rightarrow \infty$. The functions $a_{j}^{t}(x)$ satisfy the coupled equations

$$
\left(\frac{d^{2}}{d x^{2}}+2 i \frac{d}{d x}-\frac{l_{j}\left(l_{j}+1\right)}{x^{2}}\right) a_{j}^{t}(x)=\sum_{m} \frac{k w_{j m}^{[3]} a_{m}^{t}(x)}{x^{3}}+\sum_{m} \frac{k^{3} w_{j m}^{[5]} a_{m}^{t}(x)}{x^{5}}
$$

and are chosen to have the form

$$
a_{j}^{t}(x)=\sum_{n=0}^{\infty} \frac{A_{j t}^{(n)}}{x^{n}}
$$


When substituted into Eq. 6, the recurrence relation

$$
A_{j t}^{(n+4)}=\frac{\left[(n+3)(n+4)-l_{j}\left(l_{j}+1\right)\right] A_{j t}^{(n+3)}-\sum_{m} k w_{j m}^{[3]} A_{m t}^{(n+2)}-\sum_{m} k^{3} w_{j m}^{[5]} A_{m t}^{(n)}}{2 i(n+4)}
$$

is obtained. The asymptotic behaviour of Eq. 5 is ensured if $A_{j t}^{(0)}=\delta_{j t}$. The values $A_{j t}^{(1)}$, $A_{j t}^{(2)}$ and $A_{j t}^{(3)}$ are obtained by equating the coefficients of like powers of $x$ in Eq. 6 , giving

$$
\begin{aligned}
& A_{j t}^{(1)}=\frac{i}{2} l_{j}\left(l_{j}+1\right) A_{j t}^{(0)} \\
& A_{j t}^{(2)}=\frac{\left[2-l_{j}\left(l_{j}+1\right)\right] A_{j t}^{(1)}-\sum_{m} k w_{j m}^{[3]} A_{m t}^{(0)}}{4 i}, \\
& A_{j t}^{(3)}=\frac{\left[6-l_{j}\left(l_{j}+1\right)\right] A_{j t}^{(2)}-\sum_{m} k w_{j m}^{[3]} A_{m t}^{(1)}}{6 i} .
\end{aligned}
$$

The recurrence relation for the coupled incoming solutions is the complex conjugate of Eqs. 8 and 9 .

When summed directly these series have the usual properties of asymptotic series in that the partial sums converge towards the correct result and then diverge. Padé acceleration is therefore used. Particularly suitable when dealing with matrices is the $\epsilon$-algorithm [16] which is used here. This allows the acceleration to be applied to the whole matrix of partial sums, as opposed to each individual matrix element. Furthermore a matrix inversion can be avoided at each step of the iteration by the use of the pseudo inverse of the matrix.

Our model two-channel problem is solved with diagonal and off-diagonal potential elements having the form

$$
V_{i j}(r)=V_{i j}^{[3]} /\left(a_{i j}^{[3]}+r^{3}\right)+V_{i j}^{[5]} /\left(a_{i j}^{[5]}+r^{5}\right) .
$$

If the potential strengths are too weak to form a bound state in either channel, then it is expected that the two physical poles corresponding to the first bound states will be present in the fourth quadrant of the complex $k$-plane.

However, if the couplings are truncated at $20 \mathrm{fm}$, six poles are found, as shown in figure 1 (the filled circles on the dashed line). The diagonal potential strengths are: $V_{11}^{[3]}=-160, V_{11}^{[5]}=-10, V_{22}^{[3]}=-150, V_{22}^{[5]}=-10$ and $a_{i i}^{[3]}=a_{i i}^{[5]}=2, i=1,2$. The off-diagonal strengths are given by, $V_{12}^{[3]}=-2, V_{12}^{[5]}=-13, a_{12}^{[3]}=1.5, a_{12}^{[5]}=1.0$, with the potential matrix being symmetric.

The effect of including the asymptotic region of the potential can be seen by introducing a multiplicative factor $\alpha$ to alter the asymptotic strengths $w_{j m}^{[3]}$ and $w_{j m}^{[5]}$ used when evaluating 
the asymptotic solutions for matching. This is introduced so that as $\alpha$ tends to zero, the coupled asymptotic solutions tend to the solutions of the free equation, the RiccatiHankel functions, and the poles of the S-matrix are found in the same positions as when the interactions are truncated. As $\alpha$ tends to unity the full asymptotic interaction strength is included. It is seen that in this limit two of the poles move to fixed positions while the other four artificial poles continue to move away from the real $k$ axis.

\section{THE EXTREME ADIABATIC APPROXIMATION}

The adiabatic approximation, applied to the hyperspherical harmonics technique [17], provides a means of reducing the coupled system of hyper-radial equations to a single secondorder differential equation. This is achieved by diagonalizing the effective potential part of the Hamiltonian for fixed values of the hyper-radius. The wave function is then expanded in this basis set and, under the extreme adiabatic approximation, the Schrödinger equation is reduced to a single uncoupled equation, where the potential used is the eigenvalue of the effective potential. This approximation provides a lower limit to the binding energy, and is therefore a useful starting point for locating resonance poles for the three-body problem without resorting to the full and more complicated calculations.

The phase shifts for the lowest two eigen-potentials for the $0^{+}$state of ${ }^{6} \mathrm{He}$ are shown in figure 2 (i). We have seen that there is a reasonable agreement with the appropriate phase shifts for the full coupled problem. Furthermore the lowest eigen-potential, giving rise to the repulsive phase shift, has a bound state at $-1.1 \mathrm{MeV}$ in reasonable agreement with the value $-1.0 \mathrm{MeV}$ obtained in the full coupled channels calculation.

The phase shifts are shown (thick lines) when the full long-range interaction is included by matching at $25 \mathrm{fm}$ to solutions of the radial equation when it is assumed that the potential behaves asymptotically as inverse cubic and quintic radial powers as discussed in section IV. For comparison the phase shifts when the interaction is truncated at $25 \mathrm{fm}$ are also shown (thin lines). For the $K=0$ eigen-potential there is only a significant deviation of the phase shifts at low energy, while for the $K=2$ eigen-potential a significant deviation between the phase shifts is seen at all energies indicating that the correct asymptotic treatment is more important in this case.

However the truncation has a more profound implication on the position of the resonance 
pole as shown in figure 2 (ii). Here the pole positions are shown for the cases when the long-range interaction is included and when it is truncated. Four poles are found when the long-range interaction is truncated (filled circles on the dashed line), while only one is found at $k=0.23-0.12 i \mathrm{fm}^{-1}$ if it is included (filled square). This corresponds to a position in the complex energy plane of $E_{r}=0.79-1.20 i \mathrm{MeV}$. This result is consistent with the pole position located when exterior rotation is used on the analytical region of the potential. In this case the integration can be carried out to a larger distance and the matching done using Riccati-Hankel functions.

If a multiplicative factor $\lambda$ is introduced to strengthen the interaction, then for $\lambda=1.5$ it can be seen from figure 2 (ii) that the position of the pole does not significantly depend on the asymptotic portion of the potential. However as $\lambda$ is reduced to 1.0 the effect of the long-range behaviour is seen to become more significant as the pole moves further from the real $k$ axis when the full interaction is included.

Furthermore, the three artificial poles (present when the interaction is truncated) are seen to be removed from the physically significant region of the complex plane when the asymptotic scaling parameter $\alpha=1$ incorporates the full interaction.

As $\alpha$ tends to unity, the three artificial poles are seen to move away from the real $k$ axis, their positions moving rapidly for $\alpha \approx 1$. The pole that corresponds to the first bound state, when $\lambda$ is increased, does not continue to move from the real axis but converges to a fixed position for $\alpha=1$.

\section{CONCLUSION}

Artificial S-matrix poles are created in a single channel calculation when long-range interactions are truncated. They are also present in any coupled channels calculation if long-range couplings are truncated. Including correctly the long-range nature of the interaction is shown to remove these artificial poles from the physically interesting region of the complex energy plane.

The extreme adiabatic approximation applied to the $0^{+}$state of ${ }^{6} \mathrm{He}$ shows an over-binding of the ground state. If this approximation has the same implications for the position of the resonance poles, then a full coupled channels calculation for the $0^{+}$state may be expected to produce a resonance pole with a lower limit on the width of about $2 \mathrm{MeV}$. The results of 
the full three-body calculations will be presented in a subsequent publication.

\section{Acknowledgments}

The financial support of the United Kingdom Engineering and Physical Sciences Research Council (EPSRC) in the form of Grant No. GR/J95867 and a research studentship (for J.O.S) is gratefully acknowledged.

[1] M. V. Zhukov, B. V. Danilin, D. V. Fedorov, J. M. Bang, I. J. Thompson and J.S.Vaagen Phys. Rep. 231, 151 (1993)

[2] T. Aumann et al. Phys. Rev. C. 59, 1252 (1999)

[3] A. Cobis, D. V. Fedorov and A. Jensen, Phys. Rev. Lett. 79 (1997) 2411

[4] B. V. Danilin, I. J. Thompson, J. S. Vaagen and M. V. Zhukov, Nucl. Phys. A632 (1998) 383

[5] A. Csótó, nucl-th/9807016

[6] V. I. Kukulin, V. M. Krasnopol'sky, and J. Horáček, Theory of Resonances Principles and Applications (Kluwer Academic Publishers, Dordrecht, 1989)

[7] S. T. Ma, Phys. Rev. 69, 668, (1946)

[8] S. T. Ma, Phys. Rev. 71, 195, (1947)

[9] T. Wu and T. Ohmura, Quantum Theory of Scattering (Prentice-Hall, London, 1962), p. 94 ff.

[10] V. V. Pupyshev and S. A. Rakityansky Z. Phys. A348 (1994) 227

[11] A. M. Lane and R. G. Thomas, Rev. Mod. Phys. 30, 257 (1958)

[12] B. R. Junker, Adv. At. Mol. Phys. 18, 207 (1981)

[13] W. P. Reinhardt, Ann. Rev. Phys Chem. 33, 223 (1982)

[14] Y. K. Ho, Phys. Rep. 99, 1 (1983)

[15] F. Rösel, J.Phys. G. 3, 613 (1977)

[16] P. R. Graves-Morris, Padé Approximants (The Institute of Physics, London, 1973)

[17] S. Sanyal, Few-Body Systems 4, 141 (1988) 


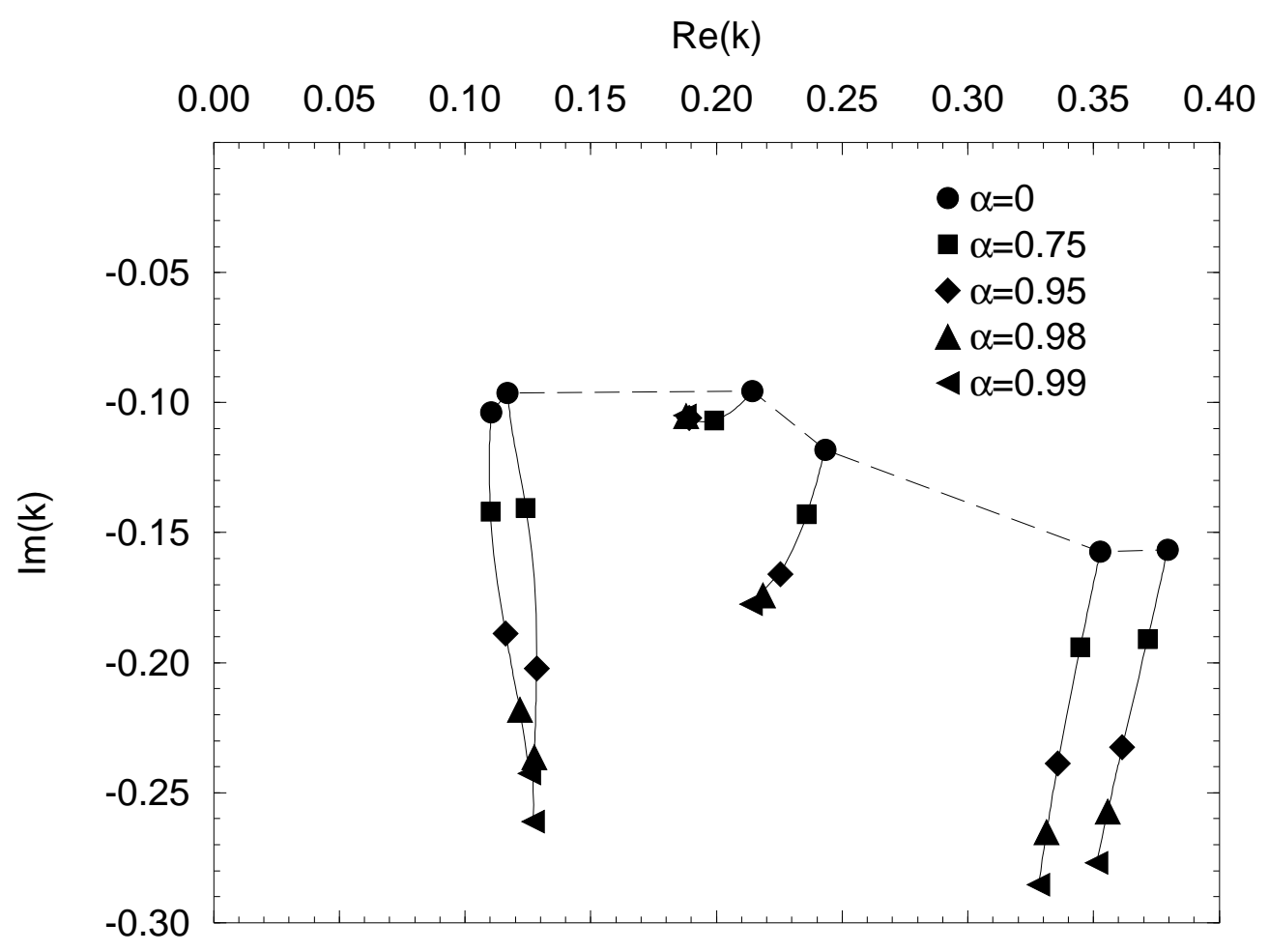

FIG. 1: A two-channel example using the values: $\hbar^{2} /(2 m)=23.223 \mathrm{MeV} \mathrm{fm}^{2}$, and $l=1$ for both channels. The artificial poles continue to move away from the real $k$ axis, as the full interaction is included, while only the two physical poles remain for $\alpha=1$. 


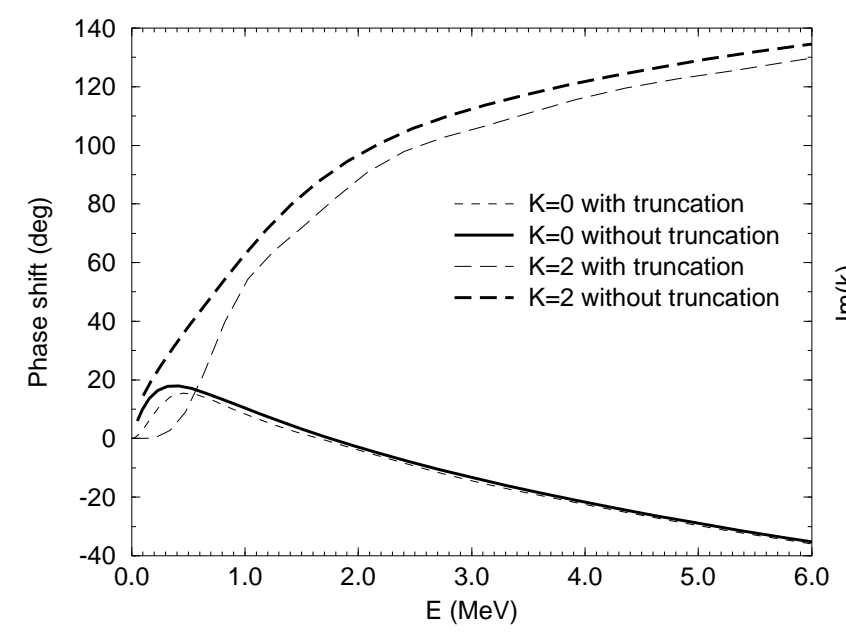

(i)

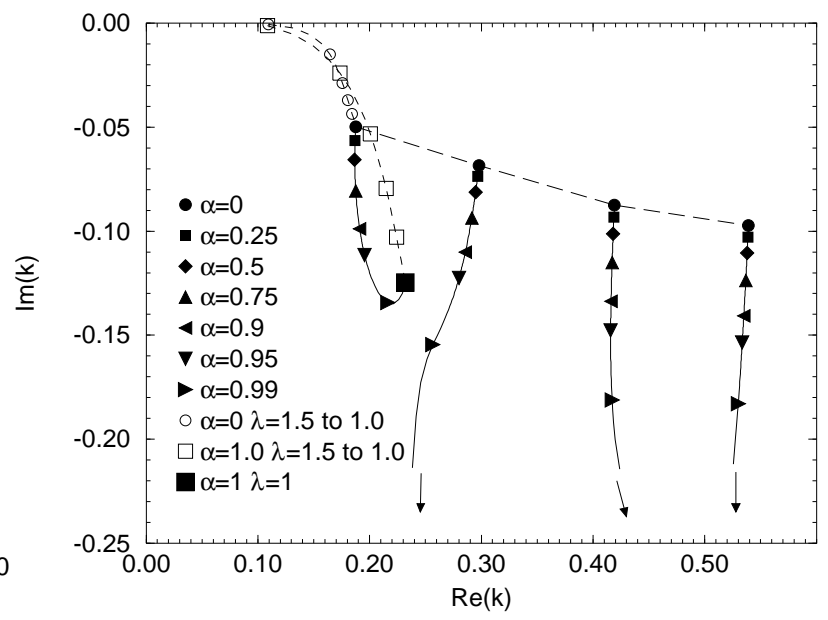

(ii)

FIG. 2: (i) Phase shifts for the lowest two eigen-potentials, $K=0$ and $K=2$, for the ${ }^{6} \mathrm{He} 0^{+}$ state when truncating the potentials at $25 \mathrm{fm}$ and also when including the appropriate asymptotic form. (ii) A multiplicative factor $\lambda$ is used to strengthen the $K=2$ eigen-potential. The pole position corresponding to the first bound state is shown when the asymptotic part of the potential is neglected (open circle) and included (open squares). The removal of the artificial poles is also shown, where the multiplicative factor $\alpha$ is used to scale the asymptotic coefficients. 